\title{
Validation of Motion Tracking Software for Evaluation of Surgical Performance in Laparoscopic Cholecystectomy
}

\author{
Sandeep Ganni ${ }^{1,2,3}$ (D) Sanne M. B. I. Botden ${ }^{4} \cdot$ Magdalena Chmarra ${ }^{1} \cdot$ Meng Li Li, $^{1,3}$ Richard H. M. Goossens ${ }^{1}$. \\ Jack J. Jakimowicz ${ }^{1,3}$
}

Received: 16 October 2019 / Accepted: 16 January 2020 Published online: 24 January 2020

(C) The Author(s) 2020

\begin{abstract}
Motion tracking software for assessing laparoscopic surgical proficiency has been proven to be effective in differentiating between expert and novice performances. However, with several indices that can be generated from the software, there is no set threshold that can be used to benchmark performances. The aim of this study was to identify the best possible algorithm that can be used to benchmark expert, intermediate and novice performances for objective evaluation of psychomotor skills. 12 video recordings of various surgeons were collected in a blinded fashion. Data from our previous study of 6 experts and 23 novices was also included in the analysis to determine thresholds for performance. Video recording were analyzed both by the Kinovea 0.8.15 software and a blinded expert observer using the CAT form. Multiple algorithms were tested to accurately identify expert and novice performances. $1 / 2 \mathrm{~L}+1 / 3 \mathrm{~A}+1 / 6 \mathrm{~J}$ scoring of path length, average movement and jerk index respectively resulted in identifying 23/24 performances. Comparing the algorithm to CAT assessment yielded in a linear regression coefficient $\mathrm{R}^{2}$ of 0.844. The value of motion tracking software in providing objective clinical evaluation and retrospective analysis is evident. Given the prospective use of this tool the algorithm developed in this study proves to be effective in benchmarking performances for psychomotor skills evaluation.
\end{abstract}

Keywords Motion tracking · Objective evaluation · Indices of performance $\cdot$ Laparoscopic skills training $\cdot$ Video-based assessment $\cdot$ Thresholds of performance

\section{Introduction}

Training and assessment in laparoscopic surgery are increasingly moving towards more objective and criterion-based evaluation tools. [1-3] Box trainers with cameras, virtual and augmented reality simulators have facilitated in achieving objective evaluation of technical skills. [4-7] Recent trends in

This article is part of the Topical Collection on Education \& Training

Sandeep Ganni

s.ganni@tudelft.nl

1 Delft University of Technology, Industrial Design Engineering, Medisign, Delft, The Netherlands

2 GSL Medical College, Department of Surgery, Rajahmundry, India

3 Catharina Hospital, Research and Education, Michelangelolaan 2, 5653 EJ Eindhoven, The Netherlands

4 Department of Pediatric Surgery, Radboudumc - Amalia Children's Hospital, Nijmegen, the Netherlands surgical training, such as self-directed learning and reflective practice, indicate a positive effect of repetitive and independent practice, which have been made possible with objective evaluation tools. [8-10] Several objective criteria such as instrument movement, procedure time, and procedure specific risky maneuvers can be extracted from these simulators and serve as benchmarks for assessing the performance or selfassessment for progress monitoring. [11, 12] However, the use of these objective criteria in the operating room to assess real surgical procedures is currently limited.

It has been proven by Yamaguchi et al. that motion tracking of the surgical instruments can objectively differentiate between expert and novice surgeons in a skills lab setting. This has been achieved using specialized instruments using motion trackers and cameras. [13-16] We have previously used a motion tracking software which is independent of specialized equipment and instruments during the procedure and can be used for retrospective performance analysis using the video recording of the procedure. [17] In this previous study three indices were identified, namely 'path length', 'sudden 
movements' and 'average movements', which could be extracted from the recorded videos classify expert and novice performances. These indices, however, were procedure specific and as such required a set of benchmarks to assess individual procedures.

Recent advances in image recognition and artificial intelligence (AI) have been proven effective in surgical skills evaluation. $[18,19]$ These systems are more task and procedure specific, because they evaluate the surgical skills required for laparoscopic knot tying, suturing or pelvic lymph node dissection. But, as with any laparoscopic surgery, skills are broadly categorized into cognitive and psychomotor skills. Cognitive skills as such are procedure specific and psychomotor skills are pan-procedural. Thus, the aim of this study is to develop a new set of benchmarks for psychomotor skills that scale between novice and expert performance and can be used in automated assessment tools.

\section{Methods}

\section{Protocol}

To determine a good threshold for the algorithm, the data has to be categorized as shown in Table 1. To determine these thresholds, the data from our previous study [17] was evaluated and recalculated. Three parameters were calculated: 'Path length' (L); 'Average distance' (A), which the instrument tip moved per time frame; and 'Number of extreme movements' $(\mathrm{J})$, defined as more than $1.0 \mathrm{~cm}$ movement per frame. If the value of the parameter was above the expert median, a score of 1 was assigned, if it was below the novice median, a score of 0 was assigned. Scores between the two medians were assigned a score between 0 and 1, scaled linearly. Following, these scores were weighted using the following equation, to create a total performance score $(\mathrm{p})$, ranging from 0 to 1 :

$w_{l}$, wand $w_{j}$, where $w_{l}+w_{a}+w_{j}={ }^{1}$ thus:

$w_{l} L+w_{a} A+w_{j} J:=\rho$

The aim of this study was to calculate the best weightings to determine expertise in uncomplicated laparoscopic cholecystectomy procedure.

First the original participant data from our previous study was used to determine the expertise thresholds as described above. [17] Following, a blinded evaluation of

Table 1 Ideal thresholding output from the algorithm

\begin{tabular}{lll}
\hline Threshold & Category & Procedures performed \\
\hline $\mathrm{p}>=2 / 3$ & Expert & 200 or more procedures \\
$\mathrm{p}<=1 / 3$ & Novice & 10 or fewer procedures \\
\hline
\end{tabular}

twelve new videos was performed by both the tracking system and the Competency Assessment Tool (CAT) for laparoscopic cholecystectomy by a blinded assessor to correlate the data. The videos were rated with the new weighting equation and evaluation for a significant correlation. These results were then compared to the previously recorded experience of the surgeon or surgical resident performing the procedure to determine whether the algorithm had correctly identified their level of psychomotor skills expertise.

\section{Participants}

This study uses data from the six 'experts' (>200 laparoscopic procedures performed) and 23 'novices' $(<10$ laparoscopic procedures performed but with a surgical background) in our previous study, to create thresholds for expertise. [17] These thresholds were then tested on an additional twelve blinded video recordings of six surgeons and six surgical residents, conducting an uncomplicated laparoscopic cholecystectomy procedure at the Catharina Hospital, Eindhoven, The Netherlands. This was to assess, by blinded trial, the ability of this thresholding algorithm in determining the psychomotor skills demonstrated in the procedure. All participants gave their consent for the video recording of the procedures used in this study and hospital ethics committee approval was obtained.

\section{Data extraction and statistics}

The tracking data of the instrument movements during the surgical procedure was extracted from the recorded videos using Kinovea 0.8.15 software. Both the thresholding calculations and extracted data were analyzed, including linear regression analysis, using MATLAB (R16b).

\section{Results}

\section{Threshold Determined}

Data from the tracking software was processed using the thresholding function and Equation described in the methods section, various weightings were evaluated and compared to the correct categorization to identify the best assessment algorithm (Table 2).

Set 5 resulted in the most correctly categorized videos, which concluded in the following Algorithm:

Assessment score $(0-1)$ : Score $=1 / 2 \mathrm{~L}+1 /{ }_{3} \mathrm{~A}+1 / 6 \mathrm{~J}$ 
Table 2 The values of the weighting parameters for the thresholding and the corresponding number of correctly identified experts and novices

\begin{tabular}{lllll}
\hline Set & Path length (L) & Average distance (A) & Extreme movements (J) & Correctly Identified \\
\hline 1 & $1 / 3$ & $1 / 3$ & $1 / 3$ & $20 / 24$ \\
2 & $1 / 3$ & $1 / 6$ & $1 / 2$ & $18 / 24$ \\
3 & $1 / 3$ & $1 / 2$ & $1 / 6$ & $19 / 24$ \\
4 & $1 / 6$ & $1 / 3$ & $1 / 2$ & $15 / 24$ \\
5 & $1 / 2$ & $1 / 3$ & $1 / 6$ & $23 / 24$ \\
6 & $1 / 6$ & $1 / 2$ & $1 / 3$ & $18 / 24$ \\
7 & $1 / 2$ & $1 / 6$ & $1 / 3$ & $21 / 24$ \\
\hline
\end{tabular}

\section{Validity of assessment algorithm}

Twelve videos were analyzed using the new algorithm with the tracking system and scored using the CAT form by a blinded expert assessor. The thresholding algorithm categorized the twelve videos as five experts, five intermediates and two novices. The expert-assigned CAT scores support this ordering as shown in Table 3. Upon unblinding the data, all the videos identified as expert videos were indeed performed by experienced surgeons and had the top four CAT scores. The other videos evaluated were in fact performances of surgical residents with an intermediate or novice level. Those identified as novices by the algorithm scored the lowest CAT score assigned by the expert assessor. One surgeon was identified as intermediate according to the algorithm, but also scored the lowest CAT score of the surgeons and had a very high jerk index.

\section{Significance level}

The CAT Tool is a comprehensive assessment tool that assesses performance across the three tasks in laparoscopic cholecystectomy in exposure of the cystic duct and artery, cystic pedicle dissection and resection of the gallbladder. [20] These tasks are further evaluated across different indices such as usage of instruments, handling of tissue, errors occurred and the end-product. For this study, we only considered the scoring across the usage of instruments and handling of tissue as they determine the psychomotor skills. Figure 1 depicts the linear regression curve plotted using the CAT score and the algorithm yielding a coefficient $\mathrm{R}^{2}$ of 0.844 .

\section{Performance scoring}

Scoring systems provide reference for ideal performance and serve as an indicator for measuring learning curve progression and consistency in performance. Upon analysis of the results from the algorithm and correlation with the CAT we propose the following range of scores as derived when using the algorithm for assessing psychomotor skills in laparoscopic cholecystectomy:

Expert performance: 0.65 and above

Intermediate performance: $0.35-0.65$

Novice performance: 0.35 and below

Table 3 The weighted score is the score calculated using the data extracted for the video and the thresholding equation, performance algorithm

\begin{tabular}{lllll}
\hline Video & Score performance algorithm & Category Identified by thresholds & CAT Score & Actual video category \\
\hline 1 & 1.00 & Expert & 21 & Surgeon \\
2 & 1.00 & Expert & 22 & Surgeon \\
3 & 1.00 & Expert & 20 & Surgeon \\
4 & 0.86 & Expert & 19 & Surgeon \\
5 & 0.67 & Expert & 20 & Surgeon \\
6 & 0.63 & Intermediate & 19 & Surgeon \\
7 & 0.54 & Intermediate & 17 & Resident \\
8 & 0.41 & Intermediate & 14 & Resident \\
9 & 0.36 & Intermediate & 14 & Resident \\
10 & 0.35 & Intermediate & 14 & Resident \\
11 & 0.09 & Novice & 13 & Resident \\
12 & 0.00 & Novice & Resident \\
\hline
\end{tabular}

Along with the category that this score yields (from Table 1). The Expert CAT score for that video is also shown and whether the video was, in fact, performed by an experienced surgeon or a student 


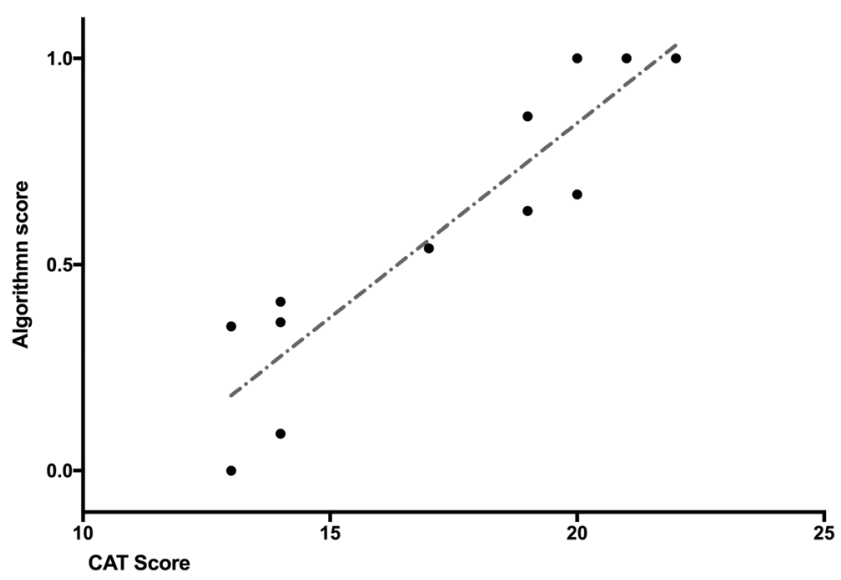

Fig. 1 Plot of Weighted score of videos, $p$ vs expert-assessed CAT score. The linear trendline has a regression coefficient of determination $\left(\mathrm{R}^{2}\right)$ of 0.844

\section{Discussion}

Traditionally assessing surgical skills requires expert assessment through standardized validated tools such as the Competency Assessment Tool (CAT) and Objective Structured Assessment of Technical Skills (OSATS) [20-22]. Objective evaluation of laparoscopic skills using motion analysis has been limited to VR simulators and robotic surgery [23]. The transfer of these evaluation criteria to clinical laparoscopic surgery has been limited by the use of additional equipment and costs [24].

Computer vision techniques and AI have shown promising results in identifying procedure specific evaluations $[18,19]$. Their strengths lie in detecting cognitive and clinical skills in addition to error recognition. AI can also effectively segment procedural steps for easy access and indexing for future reference [25]. However, these systems do not identify psychomotor skills that can be applied pan procedurally which can serve as an important indicator for learning curve monitoring in the clinical context.

Based on our previous study on the feasibility of the Kinovea software [17], the thresholds for the expertise levels were determined using results therefrom. This study was procedure-specific using uncomplicated laparoscopic cholecystectomy in the clinical setting. The thresholds were set based on a new algorithm, which was validated by comparing it with both objective expert assessors $\left(p=0.01, \mathrm{R}^{\wedge} 2=0.844\right)$. Overall, the current threshold algorithm seems to provide a potential objective assessment tool for psychomotor skills evaluation. The algorithm is weighted on the importance of each of the indices identified and the rate in which these make up the expertise of the performance.

However, this study has shown the potential value of the Kinovea tracking software to rapidly evaluate one's psychomotor skills automatically of a laparoscopic procedure, retrospectively, without the need for additional equipment during the procedure. Moreover, because the scoring is by assessing surgical videos retrospectively, there is no need for the use of other equipment or the stress of being watched by an assessor. Surgical trainees in a skills lab setting are used to objective metric scores as part of their self-improvement on VR and AR simulators and this new assessment method could be developed to act as a bridge to clinical settings; having value in both self-assessments, for improving the learning curve and as a tool for measuring psychomotor skills.

\section{Limitations}

Whilst the algorithm presents a promising first step towards bridging the gap between true objective evaluation from the skills lab to the operating theatre, the current calculations used in this study are limited in their application to assessing psychomotor skills required for laparoscopic cholecystectomy. Furthermore, as they represent a broad average of movement, these indices do not currently provide an indication of errors or potential errors. However, in combination with computer vision techniques and $\mathrm{AI}$ that are proven to recognize procedure and task specific errors based on image recognition, this algorithm could in the future be developed to serve in providing a more comprehensive evaluation of laparoscopic skills, similar to that of VR simulators, in a clinical setting. Furthermore, with the new insights of this study in the categorization of the importance of performance indices, it could be transferred to other laparoscopic procedures.

\section{Conclusion}

The value of motion tracking software in providing objective clinical evaluation and retrospective analysis is evident. Given the prospective use of this tool the algorithm developed in this study proves to be effective in benchmarking performances for psychomotor evaluation of laparoscopic skills.

\section{Compliance with Ethical Standards}

Disclosures Sandeep Ganni, Sanne MBI Botden, Magdalena K. Chmarra, Meng Li, Richard HM Goossens and Jack J. Jakimowicz have no conflicts of interest or financial ties to disclose.

Open Access This article is licensed under a Creative Commons Attribution 4.0 International License, which permits use, sharing, adaptation, distribution and reproduction in any medium or format, as long as you give appropriate credit to the original author(s) and the source, provide a link to the Creative Commons licence, and indicate if changes were made. The images or other third party material in this article are included in the article's Creative Commons licence, unless indicated otherwise in a credit line to the material. If material is not included in the article's Creative Commons licence and your intended use is not permitted by statutory regulation or exceeds the permitted use, you will need to obtain permission directly from the copyright holder. To view a copy of this licence, visit http://creativecommons.org/licenses/by/4.0/. 


\section{References}

1. Moorthy, K., Munz, Y., Sarker, S. K., and Darzi, A., Objective assessment of technical skills in surgery. Br J Surg 327(7422): 1032-1037, 2003.

2. van Hove, P. D., Tuijthof, G. J. M., Verdaasdonk, E. G. G., Stassen, L. P. S., and Dankelman, J., Objective assessment of technical surgical skills. Br J Surg, 2010. https://doi.org/10.1002/bjs.7115.

3. Oropesa et al., Methods and tools for objective assessment of psychomotor skills in laparoscopic surgery. J Surg Res, 2011. https:// doi.org/10.1016/j.jss.2011.06.034.

4. Botden, S. M. B. I., and Jakimowicz, J. J., What is going on in augmented reality simulation in laparoscopic surgery? Surg Endosc 23:1693-1700, 2008. https://doi.org/10.1007/s00464-0080144-1.

5. Bann, S., Darzi, A., Munz, Y., Kumar, B. D., and Moorthy, K., Laparoscopic virtual reality and box trainers: Is one superior to the other? Surg Endosc 18:485-494, 2004. https://doi.org/10. 1007/s00464-003-9043-7.

6. Schijven, M. P., Jakimowicz, J. J., Broeders, I. A. M. J., and Tseng, L. N. L., The Eindhoven laparoscopic cholecystectomy training course - Improving operating room performance using virtual reality training: Results from the first E.a.E.S. accredited virtual reality trainings curriculum. Surg Endosc 19(9):1220-1226, 2005. https:// doi.org/10.1007/s00464-004-2240-1.

7. Seymour, N. E., Gallagher, A. G., Roman, S. A., O'Brien, M. K., Bansal, V. K., Andersen, D. K., and Satava, R. M., Virtual reality training improves operating room performance. Ann Surg 236:458 464, 2002. https://doi.org/10.1097/00000658-200210000-00008.

8. Ganni, S., Chmarra, M. K., Goossens, R. H. M., and Jakimowicz, J. $\mathrm{J}$., Self-assessment in laparoscopic surgical skills training: Is it reliable? Surg Endosc 31(6):2451-2456, 2017.

9. Ak, G., and Adbelfattah, K., Getting better all the time? Facilitating accurate team self-assessments through simulation. BMJ Simulation and Technology Enhanced Learning, 2019. https://doi. org/10.1136/bmjstel-2018-000411.

10. Ganni, S., Botden, S. M. B. I., Schaap, D. P. et al., "Reflectionbefore-practice" improves self-assessment and end-performance in laparoscopic surgical skills training. Journal of Surgical Education, 2017. https://doi.org/10.1016/j.jsurg.2017.07.030.

11. Grantcharov, T. P., Rosenberg, J., Pahle, E., and Funch-Jensen, E., Virtual reality computer simulation - an objective method for the evaluation of laparoscopic skills. Surg Endosc, 2001. https://doi. org/10.1007/s004640090008.

12. Lamata, P., Gomez, E. J., Bello, F. et al., Conceptual framework for laparoscopic VR simulators. IEEE Comput Graph Appl 26(6):6979, 2006.

13. Yamaguchi, S., Yoshida, D., Kenmotsu, H., Yasunaga, T., Konishi, K., Ieiri, S., Nakashima, H., Tanoue, K., and Hashizume, M., Objective assessment of laparoscopic suturing skills using a motion-tracking system. Surg Endosc 25:771-775, 2010. https:// doi.org/10.1007/s00464-010-1251-3.

14. Oropesa, I., Chmarra, M. K., Sánchez-González, P., Lamata, P., Rodrigues, S. P., Enciso, S., Sánchez-Margallo, F. M., Jansen, F.-
W., Dankelman, J., and Gómez, E. J., Relevance of motion-related assessment metrics in laparoscopic surgery. Surg Innov 20:299 312, 2013. https://doi.org/10.1177/1553350612459808.

15. Hofstad, E. F., Våpenstad, C., Chmarra, M. K., Langø, T., Kuhry, E., and Mårvik, R., A study of psychomotor skills in minimally invasive surgery: What differentiates expert and nonexpert performance. Surg Endosc 27(3):854-863, 2012. https://doi.org/10.1007/ s00464-012-2524-9.

16. Ghasemloonia, A., Maddahi, Y., Zareinia, K., Lama, S., Dort, J. C., and Sutherland, G. R., Surgical skill assessment using motion quality and smoothness. Journal of Surgical Education 74(2):295-305, 2017. https://doi.org/10.1016/j.jsurg.2016.10.006.

17. Ganni, S., Botden, S. M. B. I., Chmarra, M. K., Goossens, R. H. M., and Jakimowicz, J. J., A software-based tool for video motion tracking in the surgical skills assessment landscape. Surg Endosc, 2018. https://doi.org/10.1007/s00464-018-6023-5.

18. Kowalewski, K. F., Garrow, C. R., Schmidt, M. W., Benner, L., Muller, B. P., and Nickel, F., Sensor-based machine learning for workflow detection and as key to detect expert level in laparoscopic suturing and knot-tying. Surg Endosc, 2019. https://doi.org/10. 1007/s00464-019-06667-4.

19. Baghdadi, A., Hussein, A. A., Ahmed, Y., Cavuoto, L. A., and Guru, K. A., A computer vision technique for automated assessment of surgical performance using surgeon console-feed videos. Int J Comput Assist Radiol Surg, 2018. https://doi.org/10.1007/ s11548-1881-9.

20. Miskovic, D., Ni, M., Wyles, S. M., Kennedy, R. H., Francis, N. K., Parvaiz, A., Cunningham, C., Rockall, T. A., Gudgeon, A. M., Coleman, M. G., and Hanna, G. B., Is competency assessment at the specialist level achievable? A study for the national training program in laparoscopic colorectal surgery in England. Ann Surg 257:476-482, 2013.

21. Vassilou, M. C., Feldman, L. S., Andrew, C. G., Bergman, S., Leffondre, K., Stanbridge, D., and Fried, G. M., A global assessment tool for evaluation of intraoperative laparoscopic skills. The Americal Journal of Surgery, 2004. https://doi.org/10.1016/j. amjsurg.2005.04.004.

22. Martin, J. A., Regehr, G., Reznick, R. et al., Objective structured assessment of technical skill (OSATS) for surgical residents. $\mathrm{Br} J$ Surg 84:243-278, 1997.

23. Reiley, C. E., Lin, H. C., Yuh, D. D. et al., Review of methods for objective surgical skill evaluation. Surg Endosc 25:356, 2011. https://doi.org/10.1007/s00464-010-1190-z.

24. Chmarra, M. K., Grimbergen, C. A., and Dankelman, J., Systems for tracking minimally invasive surgical instruments. Min Inv Ther All Tech 16(6):328-340, 2007.

25. Hashimoto, D. A., Rosman, G., Volkov, M., Rus, D. L., and Meireles, O. R., Artificial intelligence for intraoperative video analysis: Machine Learning's role in surgical education. $J$ Am Coll Surg, 2017. https://doi.org/10.1016/j.jamcollsurg.2017.07.387.

Publisher's Note Springer Nature remains neutral with regard to jurisdictional claims in published maps and institutional affiliations. 